\title{
Effectiveness of active school transport interventions: a systematic review and update
}

\author{
Richard Larouche ${ }^{1,2}$, George Mammen ${ }^{3}$, David A. Rowe ${ }^{4}$ and Guy Faulkner ${ }^{5,6^{*}}$ (D)
}

\begin{abstract}
Background: Active school transport (AST) is a promising strategy to increase children's physical activity. A systematic review published in 2011 found large heterogeneity in the effectiveness of interventions in increasing AST and highlighted several limitations of previous research. We provide a comprehensive update of that review.

Methods: Replicating the search of the previous review, we screened the PubMed, Web of Science, Cochrane, Sport Discus and National Transportation Library databases for articles published between February 1, 2010 and October 15, 2016. To be eligible, studies had to focus on school-aged children and adolescents, include an intervention related to school travel, and report a measure of travel behaviors. We assessed quality of individual studies with the Effective Public Health Practice Project quality assessment tool, and overall quality of evidence with the Grades of Recommendation, Assessment, Development, and Evaluation (GRADE) approach. We calculated Cohen's $d$ as a measure of effect size.

Results: Out of 6318 potentially relevant articles, 27 articles reporting 30 interventions met our inclusion criteria. Thirteen interventions resulted in an increase in AST, 8 found no changes, 4 reported inconsistent results, and 5 did not report inferential statistics. Cohen's $d$ ranged from -0.61 to 0.75 , with most studies reporting "trivial-to-small" positive effect sizes. Three studies reported greater increases in AST over longer follow-up periods and two Safe Routes to School studies noted that multi-level interventions were more effective. Study quality was rated as weak for 27/30 interventions (due notably to lack of blinding of outcome assessors, unknown psychometric properties of measurement tools, and limited control for confounders), and overall quality of evidence was rated as low. Evaluations of implementation suggested that interventions were limited by insufficient follow-up duration, incomplete implementation of planned interventions, and limited access to resources for low-income communities.
\end{abstract}

Conclusions: Interventions may increase AST among children; however, there was substantial heterogeneity across studies and quality of evidence remains low. Future studies should include longer follow-ups, use standardized outcome measures (to allow for meta-analyses), and examine potential moderators and mediators of travel behavior change to help refine current interventions.

Trial registration: Registered in PROSPERO: CRD42016033252

Keywords: Active travel, Physical activity, Children, Safe routes to school, School travel plans, Walking school buses

\footnotetext{
* Correspondence: guy.faulkner@ubc.ca

${ }^{5}$ School of Kinesiology, University of British Columbia, D H Copp Building

4606, 2146 Health Sciences Mall, Vancouver, BC V6T 1Z3, Canada

${ }^{6}$ Center for Hip Health and Mobility, Robert H.N. Ho Research Centre, 5th

Floor, 2635 Laurel St, Vancouver, BC V5Z 1M9, Canada

Full list of author information is available at the end of the article
}

(c) The Author(s). 2018 Open Access This article is distributed under the terms of the Creative Commons Attribution 4.0 International License (http://creativecommons.org/licenses/by/4.0/), which permits unrestricted use, distribution, and reproduction in any medium, provided you give appropriate credit to the original author(s) and the source, provide a link to the Creative Commons license, and indicate if changes were made. The Creative Commons Public Domain Dedication waiver (http://creativecommons.org/publicdomain/zero/1.0/) applies to the data made available in this article, unless otherwise stated. 


\section{Background}

Consistent evidence shows that children and adolescents who engage in active school transport (AST) are more physically active than those who travel by motorized vehicles [1, 2]. Cycling to and from school can also increase cardiovascular fitness [1] and is associated with a better cardiometabolic health profile [3]. At the population level, replacing motorized travel by AST could reduce exhaust and greenhouse gas emissions [4, 5]. Additional benefits of AST include positive emotions during the school trip [6], better way-finding skills [7] and superior school grades [8].

Despite these benefits, the prevalence of AST has decreased markedly during the last few decades in many countries [9-13]. To address this issue, many interventions have been implemented. Perhaps the most wellknown is the Safe Routes to School (SRTS) program which has received over one billion dollars in funding from the US government [14]. Recent analyses concluded that New York City's SRTS program led to a $33-44 \%$ reduction in injuries among school-aged children and the program was cost-effective even when disregarding any potential benefits related to increased physical activity and decreased congestion and pollution $[15,16]$. In other jurisdictions, school travel plans (STP) have been implemented to address key barriers to AST at the local level, but often with limited funding [17-21]. Moreover, walking school buses (WSB) wherein children walk together on a set route with adult supervision have been implemented in many jurisdictions to address parental safety concerns [22, 23].

To our knowledge, Chillón and colleagues [24] published the first systematic review of the effectiveness of AST intervention among children and adolescents in 2011. While the included interventions were quite heterogeneous, most observed small increases in AST. However, quality of evidence for all interventions were rated as "weak" based on the Effective Public Health Practice Project (EPHPP) quality assessment tool for quantitative studies [25]. Moreover, none of the interventions examined the moderators and mediators of travel behavior change. A better understanding of moderators and mediators would enable researchers to understand what works for whom and why. We provide a comprehensive update on the effectiveness of AST interventions in children and youth that have been published over the last 6 years. We also aimed to review the literature on the moderators and mediators of AST interventions.

\section{Methods}

\section{Search strategy}

As our goal was to update the previous review [24], we replicated their search strategy. Databases searched included PubMed, Web of Science (SCI and SSCI),
SPORTDiscus, the Cochrane library, and the National Transportation Library. The search terms addressed four main categories: school-age children (adolescen* OR child OR children OR youth OR student* OR pupil OR pupils) AND active transportation (bike OR bikers OR biking OR bicycl* OR cycle OR cycling OR cyclist* OR commute* OR commuting OR transportation OR travel*) AND intervention (intervention* OR implement* OR evaluat* OR change OR pilot OR project OR environment* OR engineer* $^{*}$ OR encourage* OR planning OR impact OR "walk to school" OR "safe routes to school" OR "walking schoolbus" OR "walking school bus" OR "walking school buses") AND school. Articles published between February 1, 2010 (the cut-off date of the previous review) and October 15, 2016 were considered eligible. Our review is registered in PROSPERO (CRD42016033252; see http://www.crd.york.ac.uk/PROSPERO/display_record.asp? ID=CRD42016033252).

\section{Inclusion and exclusion criteria}

To be included in the review, studies had to: 1) have been conducted among children and adolescents (6-18 year olds); 2) focus on AST; 3) include an intervention; and 4) examine the effect of the intervention on a measure of active transportation or physical activity. Studies that did not meet all of these criteria were excluded. Language was not an exclusion criterion. Titles and abstract of all potentially relevant articles were screened by GM and GF. Full text copies of all articles that were not excluded at this stage of the review were then screened by GM and GF. Any discrepancy was resolved by consensus.

\section{Data extraction}

The following data were extracted from each included study: lead author, country, a brief description of the intervention and its methodology, the effects on AST, the moderators and mediators examined, the effects on other outcomes and the types of strategies that were used based on the Safe Routes to School 6E model [14]. The 6 E's are: 1) education (teaching students and community members about the different transportation options and ensuring they have the skills and know-how to be safe in traffic); 2) encouragement (using events, activities and incentives to promote AST); 3) engineering (making improvements to the built environment to increase safety); 4) enforcement (partnering with law enforcement to address traffic and crime concerns in the neighborhoods around schools and along school routes); 5) evaluation (assessing the effectiveness of the interventions); and 6) equity (ensuring that initiatives are benefiting all demographic groups). By definition, all studies that met our inclusion criteria have used evaluations, so this strategy was not extracted. Data extraction was done by RL and GM for a subsample of studies, and only by 
RL for the remainder. When relevant information was missing from included papers, we attempted to contact the lead author and/or the senior author.

\section{Quality assessment}

To assess the methodological quality of each study, we used an adapted version of the EPHPP. This tool includes 6 components: 1) selection bias; 2 ) study design; 3 ) control for confounders; 4) blinding of participants and study staff; 5) validity and reliability of the data collection tools; and 6) withdrawals and drop-outs. Each component was rated as "weak", "moderate" or "strong" based on standardized criteria, and then the number of weak ratings was tallied. Following the EPHPP approach, studies with zero weak ratings were rated as strong, studies with one weak rating were rated as moderate, and studies with at least two weak ratings were rated as weak. We retained the modifications proposed by Chillón and colleagues [24] to make the tool more suitable to studies in which the school is the unit of allocation. We also added a number of precisions to clarify the interpretation of the items. Our adapted EPHPP tool is available in Additional file 1. Quality assessment was first performed by RL and DR for a subsample of five studies. After consensus was attained for these studies, the remaining articles were assessed either by RL or DR. In case of doubt, the reviewer was asked to indicate the issue in an Excel spreadsheet and all issues were resolved by consensus among the two reviewers. Because blinding of participants was considered unfeasible in the context of most AST interventions, we present results both with and without the blinding component of the EPHPP. In addition, we assessed overall quality of evidence using the "Grades of Recommendation, Assessment, Development, and Evaluation" (GRADE) approach [26, 27]. Following this approach, randomized controlled trials begin as high quality evidence, but they may be downgraded based on limitations in the design and implementation, indirectness of evidence, unexplained heterogeneity of results, imprecision of estimates, and high probability of publication bias. Observational studies begin as low quality evidence, but may be upgraded if there are large effect sizes, a dose-response gradient, or if all plausible confounding would reduce the treatment effect $[26,27]$. The overall quality of evidence was rated by consensus among the authors.

\section{Statistical analyses}

Following the procedures of Chillón and colleagues [24], we computed Cohen's $d$ as a measure of effect size for each intervention. For interventions that included a control group, effect size was computed as the standardized mean difference of the changes in AST between the experimental and control groups. For those that included only an experimental group, it was computed between baseline and follow-up data. Additional file 2 provides comprehensive details on how effect sizes were computed for each intervention. Authors were contacted to obtain information required to calculate $d$. Following Cohen's [28] guidelines, effect size was categorized as trivial $(d<0.2)$, small $(d=0.2)$, medium $(d=0.5)$, or large $(d=0.8)$. Due to the large methodological heterogeneity of the included studies (see Table 1), meta-analysis was considered inappropriate.

\section{Results}

The flow of papers in the review process is depicted in Fig. 1. Overall, 6318 papers were identified by the search including 2339 in PubMed, 1555 in Web of Science, 377 in Cochrane, 882 in SPORTDiscus, and 1165 in the National Transportation Library. One paper was identified from the authors' personal libraries. All abstracts were screened, and 54 papers were found to be potentially eligible for inclusion. After a thorough selection process, 27 papers were excluded due to the following exclusion criteria: no/ineligible intervention, $n=17$; no measure of physical activity or AST, $n=8$; review article, $n=2$. A total of 27 papers, reporting on 30 different interventions, were included for analyses [17-20, 29-51]. Results are presented at the intervention level because three papers reported the findings of two different interventions. Specifically, Buckley et al. [29] included a fall event without control group and a spring event with control group, Crawford and Garrard [34] included a pilot study with control schools (pilot schools) and a main study without control group (program schools), and Johnson et al. [41] reported case-control analyses using data from two different surveys conducted in distinct populations (Bikeability and CensusAtSchool). Eleven interventions were conducted in the US, five in the UK, three in Canada, two in Australia, Belgium, Denmark and New Zealand, and one in Spain and China. Another intervention was conducted simultaneously in Canada and the UK.

\section{Characteristics of interventions}

Of these interventions, six evaluated Safe Routes to School (SRTS) interventions [38, 39, 42, 43, 46, 48], seven evaluated school travel plan (STP) projects [17-20, 30, 34], and two examined stand-alone walking school buses (WSB) schemes [45, 47]. Four interventions focused on the effects of bicycle training programs [35, 36, 41], five examined the effects of stand-alone events or contests $[29,31,33,40]$, and two were multi-component interventions that examined, among other things, changes in AST following the intervention [32, 51]. Others included two studies examining the effect of curriculum-based programs on AST $[44,50]$, one intervention using a drop-off spot from which driven children could walk to school with adult supervision [49], and an investigation of the effect of 


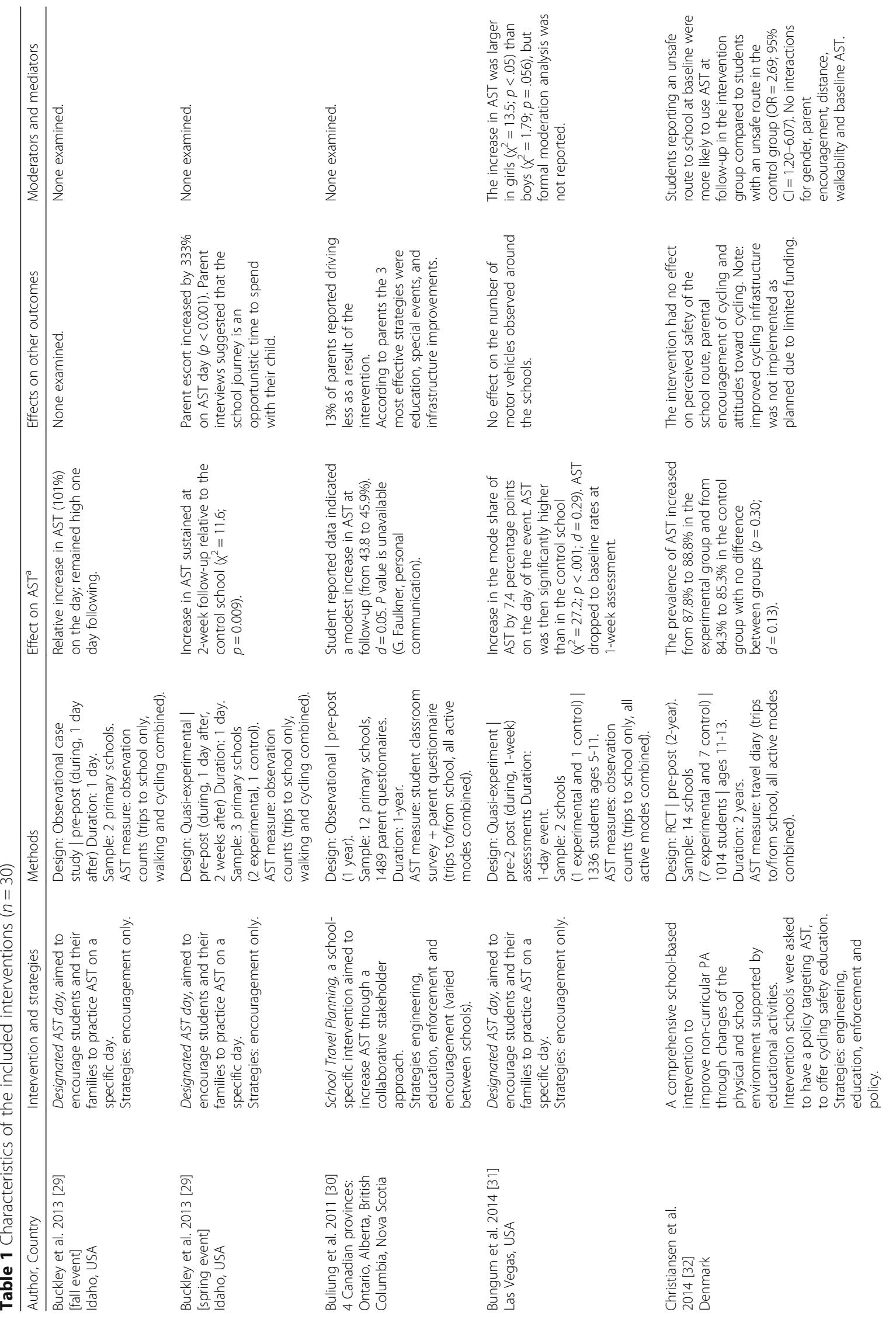




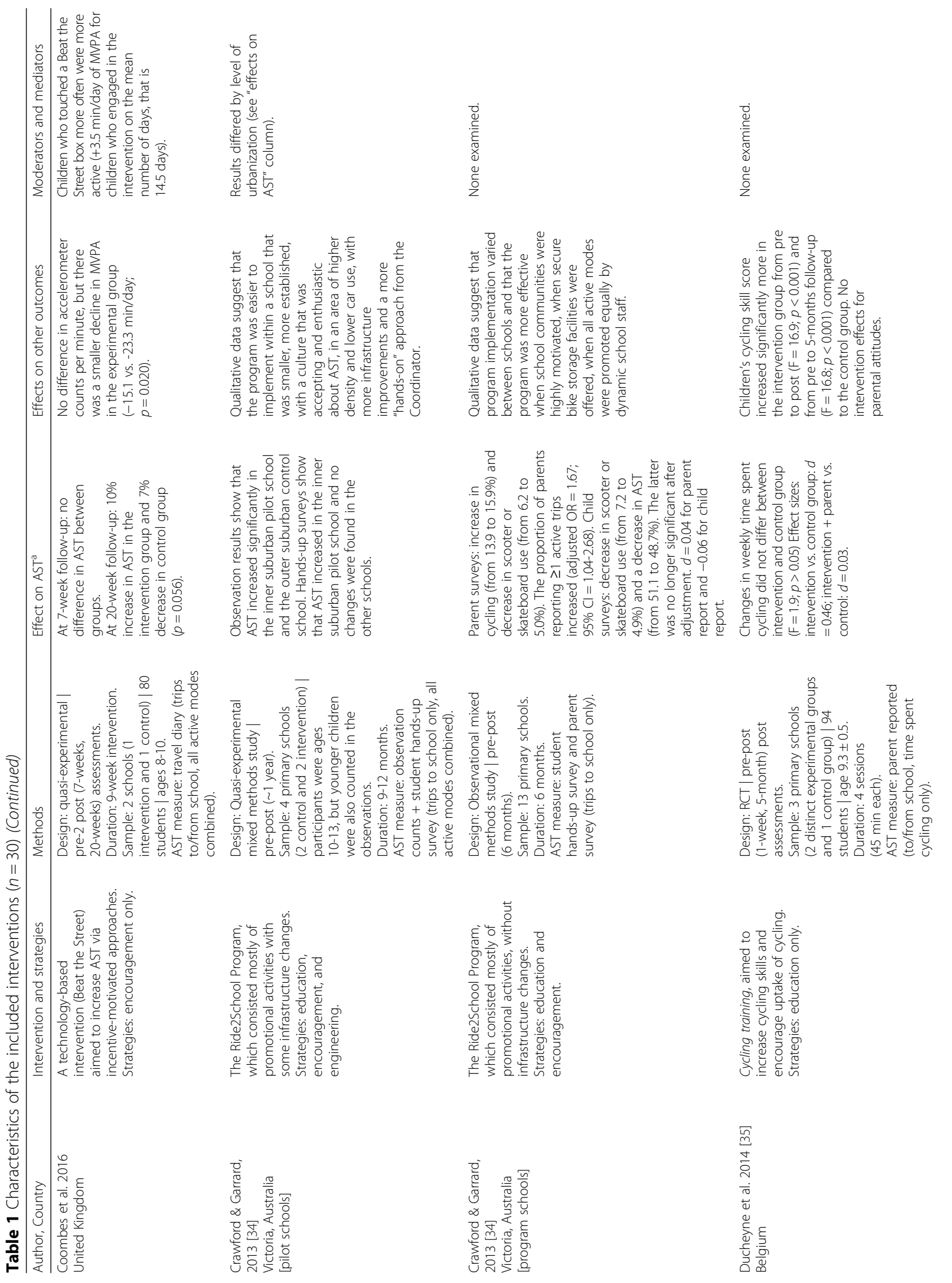




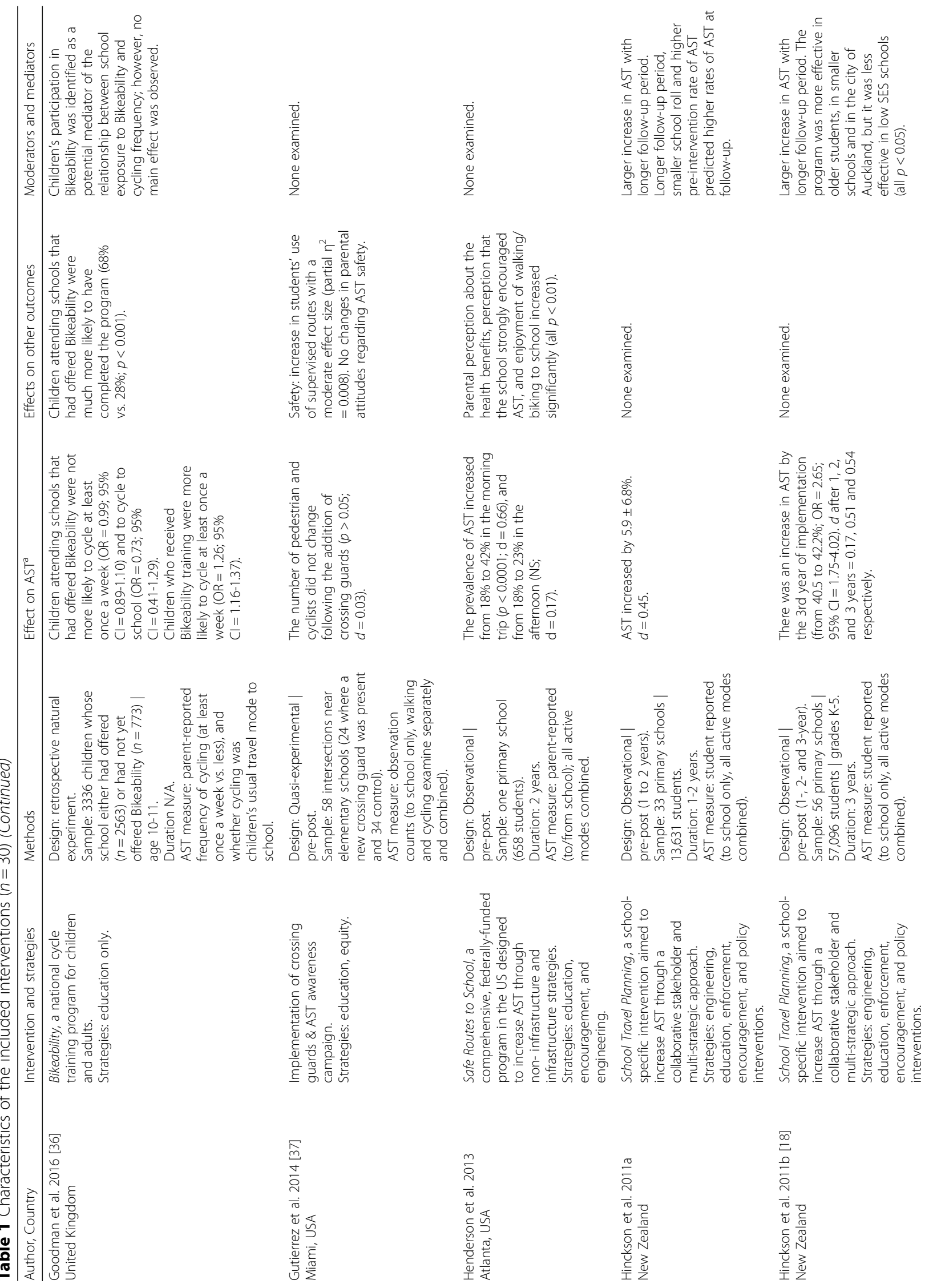




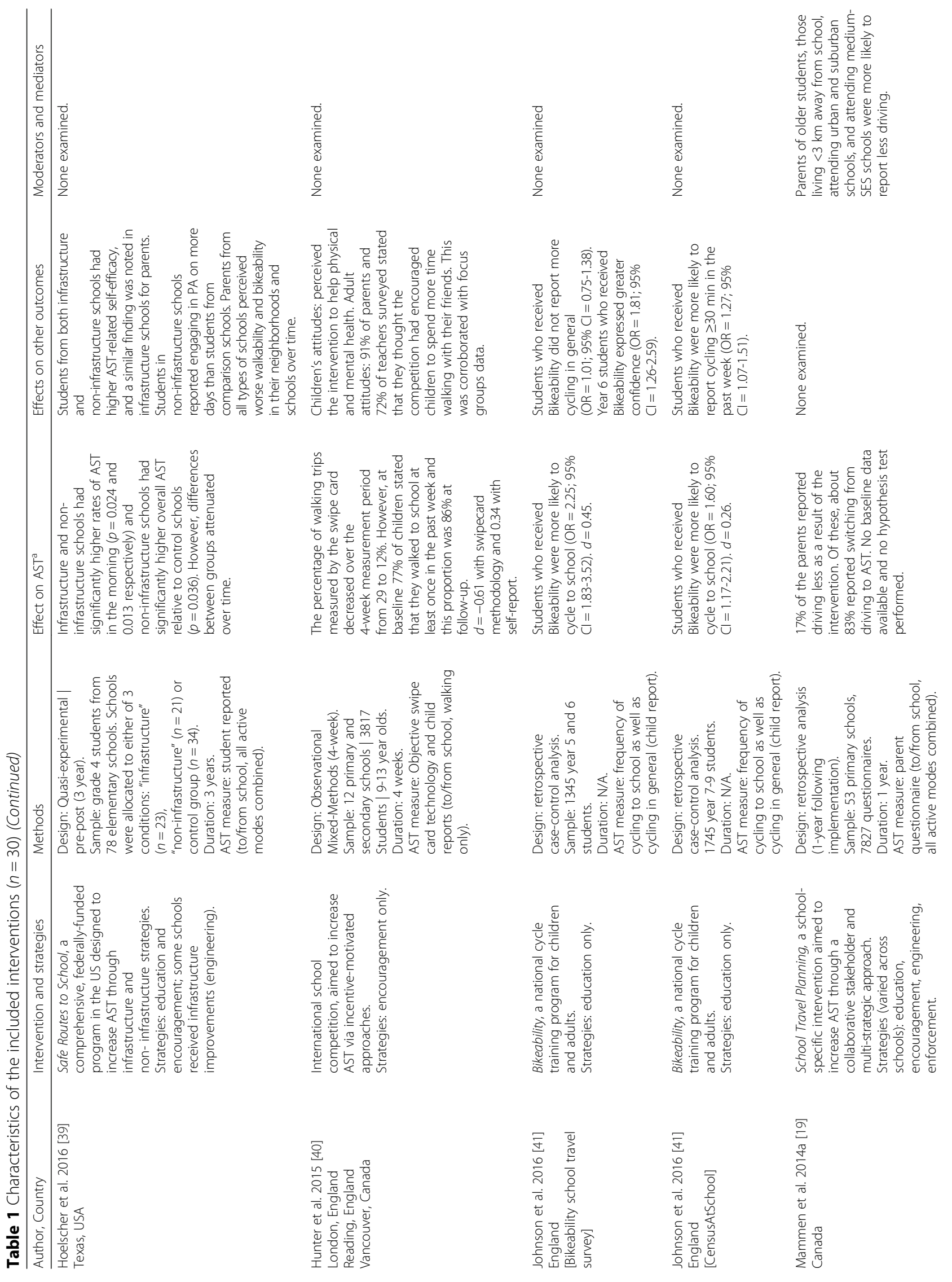




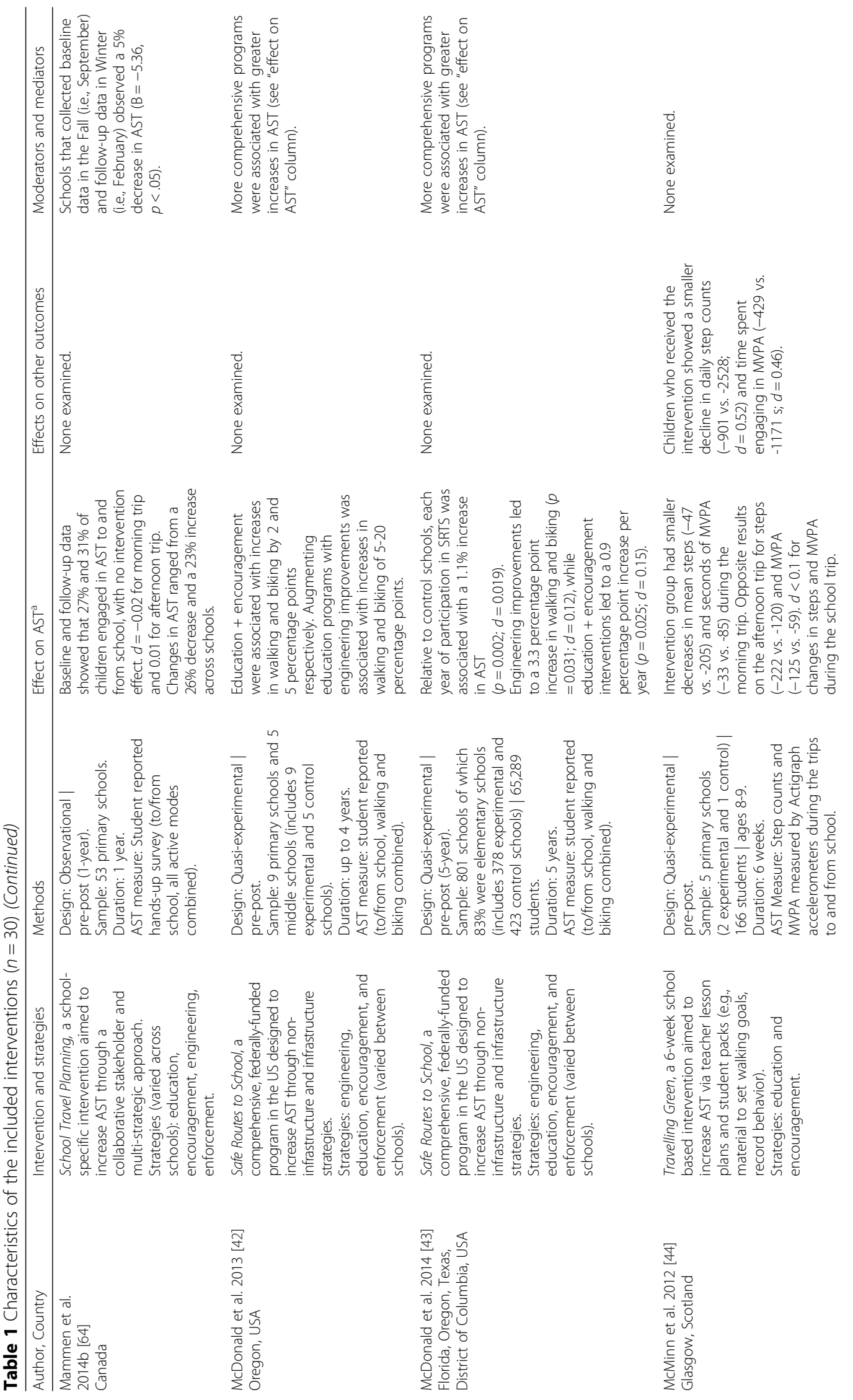




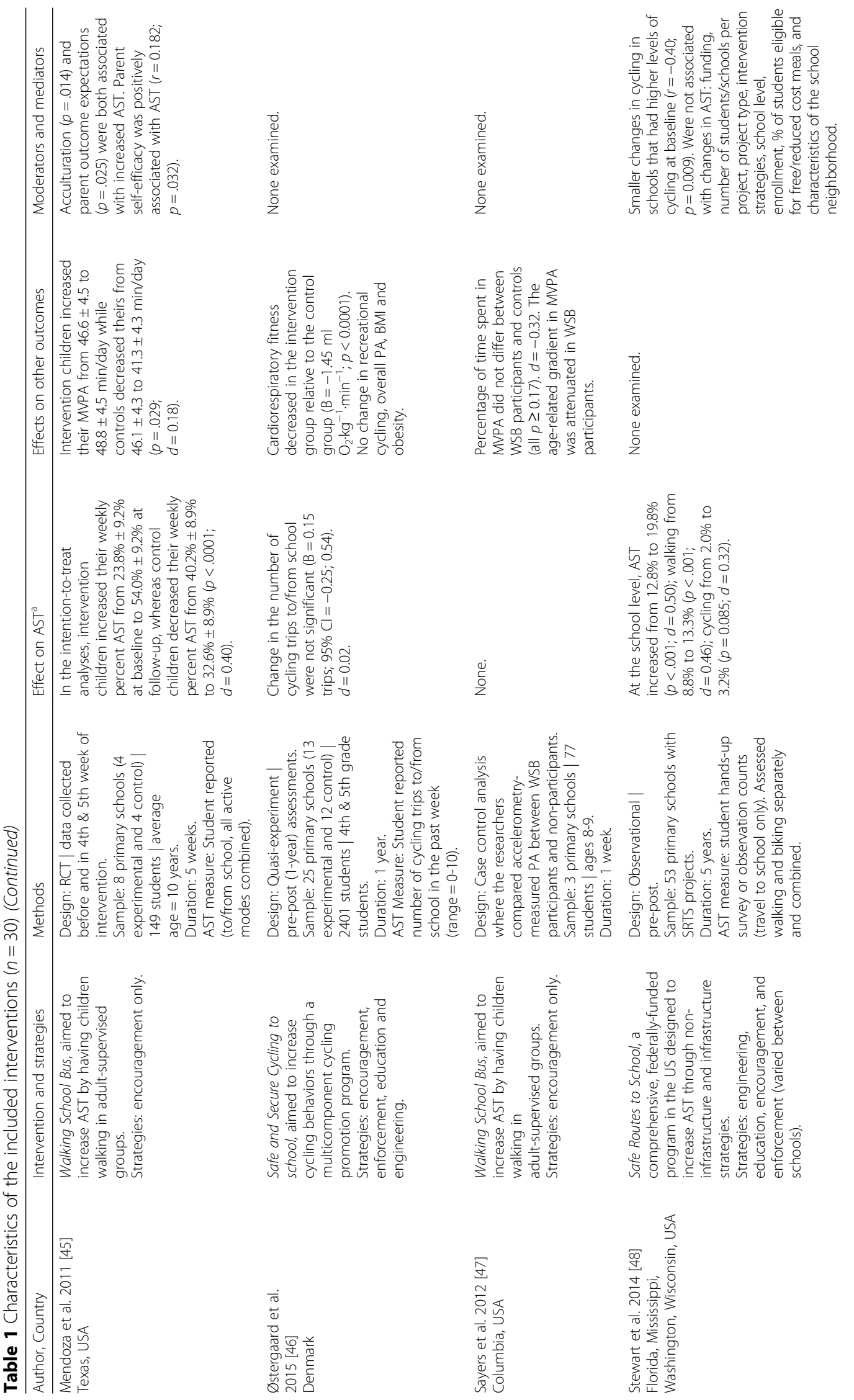




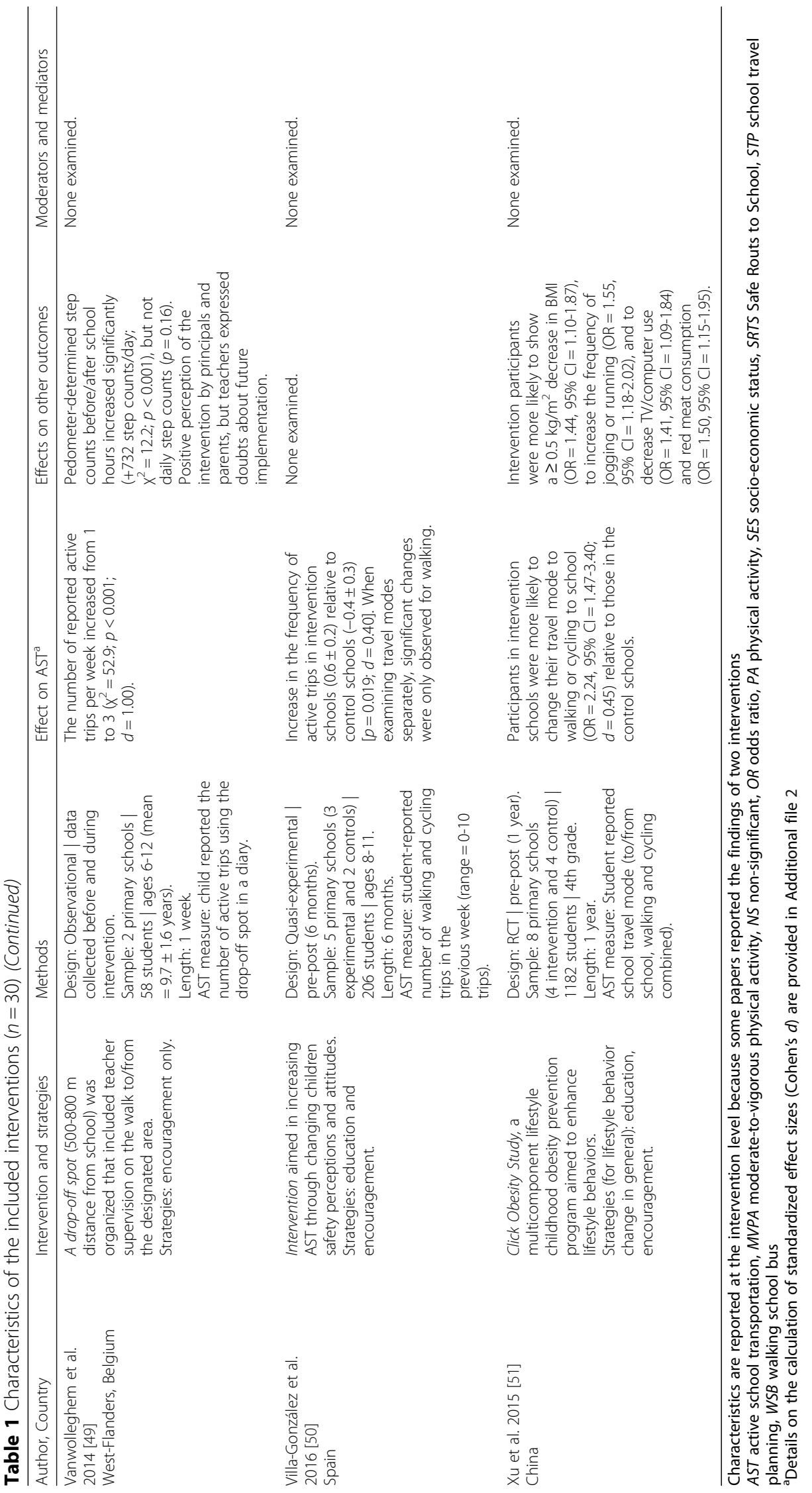




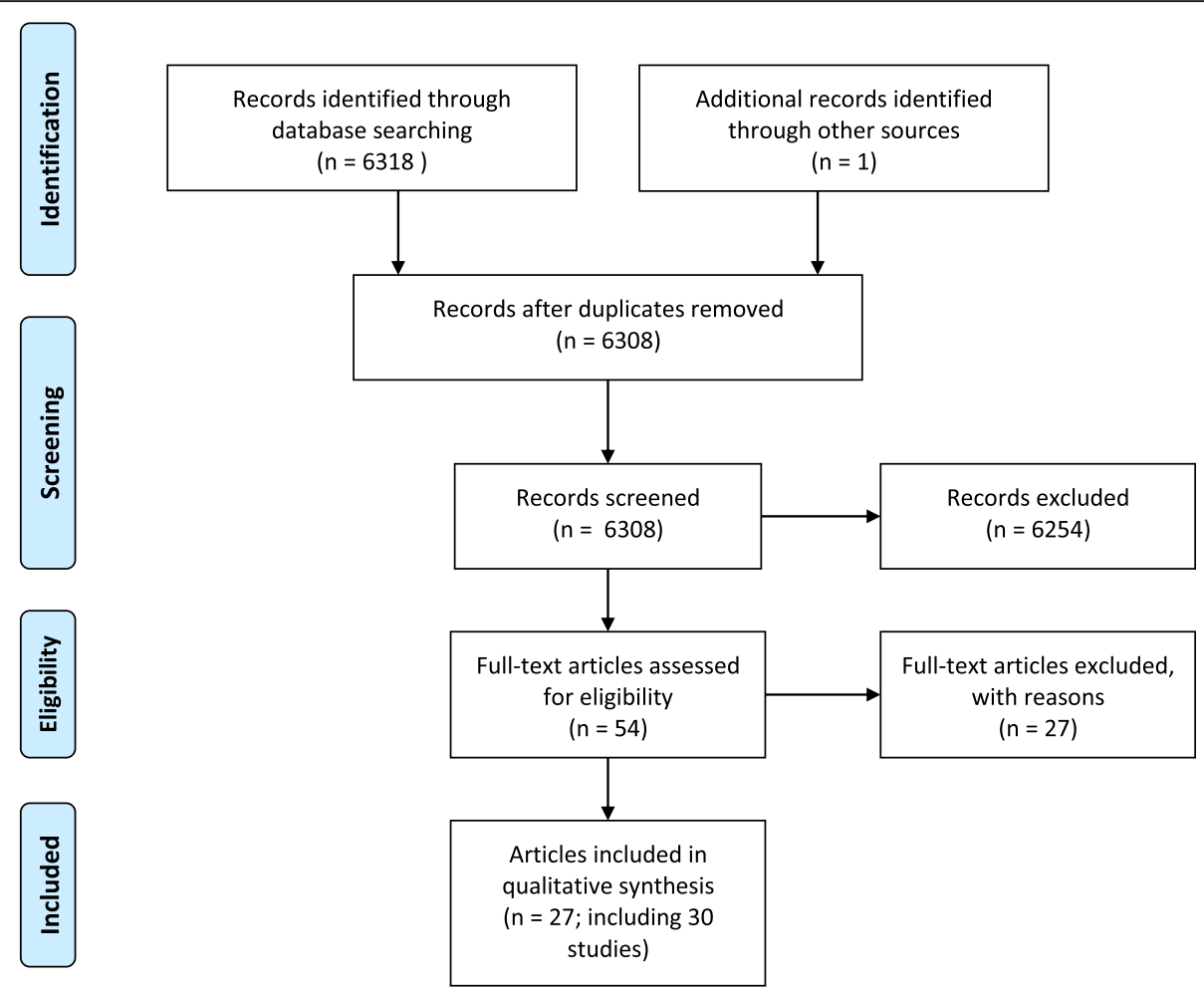

Fig. 1 Flow of articles in the review process

deploying crossing guards on travel behaviors [37]. Included studies assessed AST in a variety of ways including classroom hand-up surveys [17, 18, 20, 34, 42, 43, 48], child surveys and diaries [32, 33, 41, 45, 46, 49-51], parent surveys [19, 30, 34-36, 38], direct observation [29, 31, 32, 37, 48], using a swipe card technology [40] or by recording accelerometer steps taken during the school journey [44]. One study compared accelerometrymeasured PA among participants in a WSB and nonparticipants [47]. Moreover, there was substantial heterogeneity in how AST was operationalized (e.g., travel mode on the day of the survey, usual travel mode, frequency of AST, etc.) and whether different active modes were assessed separately or pooled together (Table 1).

The majority of interventions focused on the elementary school setting. Only three studies included some secondary school students $[40,41,43]$.The target sample size of included interventions ranged from 80 to 65,289 students. Schools were randomized to an intervention or a control group in four interventions [32, 35, 45, 51]. Of the remaining interventions, 11 used a pre-post design without a control group [17, 18, 20, 29, 30, 34, 38-40, 48, 49], 10 were quasi-experimental studies with a control group $[29,31,33,34,37,42-44,46,50]$, four were retrospective case-control studies [36, 41, 47], and one was a retrospective study [19]. A detailed description of the interventions and their main results is provided in Table 1.

\section{Quality assessment}

Quality ratings are shown in Table 2. For individual components of the EPHPP, the proportion of weak ratings was $3.3 \%$ for study design, $30.0 \%$ for withdrawals and dropouts, $56.7 \%$ for selection bias, $60.0 \%$ for control for confounders, $66.7 \%$ for data collection methods, and $100 \%$ for blinding. Following Chillón and colleagues' [24] modifications of the EPHPP, four studies were rated "non-applicable" for withdrawals and dropouts because participants were recruited after the intervention occurred and could not have dropped out. No study reported that outcome assessors or participants were blinded, and only two studies discussed blinding and specified that it was not feasible in their intervention $[35,45]$. In analyses that included the blinding component of the EPHPP tool, only three studies were rated as "moderate" [32, 39, 45], and the remainder were rated as "weak". In a sensitivity analysis that excluded the blinding component, study quality was rated as weak for 21 interventions [19, 20, 29-31, 33, 34, 36, 38, 40-43, 46, 48-51], moderate for six interventions [17, 18, 35, 37, 44, 47], and strong for three interventions [32, 39, 45]. While our review included some randomized controlled trials, most individual studies were rated as "weak" and very serious limitations in the design and implementation of interventions were noted, as mentioned above. Therefore, we attributed a low grade for the overall quality of evidence. 
Table 2 Quality assessment of active school transportation interventions

\begin{tabular}{|c|c|c|c|c|c|c|c|c|}
\hline Lead author (year) & Selection bias & $\begin{array}{l}\text { Study } \\
\text { design }\end{array}$ & $\begin{array}{l}\text { Control for } \\
\text { confounders }\end{array}$ & Blinding & $\begin{array}{l}\text { Data } \\
\text { collection }\end{array}$ & $\begin{array}{l}\text { Withdrawals } \\
\text { and dropouts }\end{array}$ & $\begin{array}{l}\text { Global } \\
\text { rating }\end{array}$ & $\begin{array}{l}\text { Global rating } \\
\text { without blinding }\end{array}$ \\
\hline Buckley (2013) [fall event] & Weak & Moderate & Weak & Weak & Weak & Strong & Weak & Weak \\
\hline Buckley (2013) [spring event] & Weak & Moderate & Strong & Weak & Weak & Strong & Weak & Weak \\
\hline Buliung (2011) & Weak & Moderate & Weak & Weak & Weak & Weak & Weak & Weak \\
\hline Bungum (2014) & Weak & Moderate & Weak & Weak & Weak & Strong & Weak & Weak \\
\hline Christiansen (2014) & Strong & Strong & Strong & Weak & Moderate & Moderate & Moderate & Strong \\
\hline Coombes (2016) & Weak & Moderate & Weak & Weak & Weak & Strong & Weak & Weak \\
\hline Crawford (2013) [pilot] & Weak & Strong & Strong & Weak & Weak & Strong & Weak & Weak \\
\hline Crawford (2013) [program] & Weak & Moderate & Weak & Weak & Weak & Strong & Weak & Weak \\
\hline Ducheyne (2014) & Moderate & Strong & Strong & Weak & Weak & Strong & Weak & Moderate \\
\hline Goodman (2016) & Moderate & Moderate & Strong & Weak & Weak & Weak & Weak & Weak \\
\hline Gutierrez (2014) & Moderate & Strong & Strong & Weak & Weak & Strong & Weak & Moderate \\
\hline Henderson (2013) & Moderate & Moderate & Weak & Weak & Weak & Weak & Weak & Weak \\
\hline Hinckson (2011a) & Moderate & Moderate & Weak & Weak & Moderate & Moderate & Weak & Moderate \\
\hline Hinckson (2011b) & Moderate & Moderate & Weak & Weak & Moderate & Strong & Weak & Moderate \\
\hline Hoelscher (2016) & Moderate & Moderate & Strong & Weak & Strong & Strong & Moderate & Strong \\
\hline Hunter (2015) & Weak & Moderate & Weak & Weak & Weak & Weak & Weak & Weak \\
\hline Johnson (2016) [Bikeability] & Weak & Moderate & Weak & Weak & Weak & N/A & Weak & Weak \\
\hline Johnson (2016) [CensusAtSchool] & Weak & Moderate & Weak & Weak & Weak & N/A & Weak & Weak \\
\hline Mammen (2014a) & Weak & Weak & Weak & Weak & Weak & N/A & Weak & Weak \\
\hline Mammen (2014b) & Moderate & Moderate & Weak & Weak & Strong & Weak & Weak & Weak \\
\hline McDonald (2013) & Weak & Moderate & Weak & Weak & Moderate & Weak & Weak & Weak \\
\hline McDonald (2014) & Weak & Moderate & Strong & Weak & Weak & Weak & Weak & Weak \\
\hline McMinn (2012) & Moderate & Moderate & Weak & Weak & Strong & Strong & Weak & Moderate \\
\hline Mendoza (2011) & Moderate & Strong & Strong & Weak & Strong & Strong & Moderate & Strong \\
\hline Østergaard (2015) & Weak & Moderate & Weak & Weak & Weak & Moderate & Weak & Weak \\
\hline Sayers (2012) & Weak & Moderate & Strong & Weak & Strong & N/A & Weak & Moderate \\
\hline Stewart (2014) & Moderate & Moderate & Weak & Weak & Weak & Weak & Weak & Weak \\
\hline Vanwolleghem (2014) & Weak & Moderate & Weak & Weak & Strong & Strong & Weak & Weak \\
\hline Villa-Gonzalez (2016) & Moderate & Moderate & Strong & Weak & Weak & Weak & Weak & Weak \\
\hline Xu (2015) & Weak & Strong & Strong & Weak & Weak & Strong & Weak & Weak \\
\hline
\end{tabular}

Quality assessment was conducted with a modified version of the Effective Public Health Practice Project quality assessment tool for quantitative studies (EPHPP, 2003), which is provided in Additional file 1. Following EPHPP guidelines, studies with no weak ratings are rated "strong", studies with one weak rating are rated "moderate" and studies with more than one weak rating are rated "weak". Considering that blinding of participants may not be feasible in the context of AST interventions, global ratings with and without the blinding component of the EPHPP are presented

\section{Intervention effectiveness}

Overall, 13 interventions resulted in a statistically significant increase in AST [18, 29, 31, 38, 41, 42, 45, 47-51] while eight reported no changes in AST [20, 32, 33, 35, 37, 43, 46, 47]. Of the latter studies, McMinn et al. [43] reported a smaller seasonal decline in PA among children in their intervention group, and this can be viewed as a positive finding given that PA typically declines during the fall and winter. Five interventions did not include an hypothesis test for changes in AST [17, 19, 29, 30, 40]. The remaining studies reported inconsistent or conflicting results. Specifically, in their pilot study, Crawford \& Garrard [34] reported a significant increase in AST in their inner suburban school, but no change in their outer suburban school relative to the control group. In their "program" phase, they reported a significant increase in AST in experimental schools based on parent surveys after adjusting for confounders, but their child surveys indicated no change in AST after statistical adjustment. Goodman and colleagues [36] reported that children attending a school that had offered the Bikeability program did not cycle more frequently; however, those who actually took part in Bikeability did cycle more frequently, suggesting that parents/children interested in cycling may have self-selected to participate. 
Finally, Hoelscher et al. [39] observed that while intervention schools had higher rates of AST over the 4-year study period, the differences between groups waned over time.

Details on the computation of effect sizes (Cohen's $d$ ) are provided in Additional file 2. Cohen's $d$ varied markedly across interventions with a range of -0.61 to 0.75 . Effect size could not be calculated for five interventions, including two that provided only follow-up data $[19,42]$, and three that provided insufficient data to allow for computation of $d$ [29,39]. Effect size was rated as trivial for 10 interventions [17, 20, 30, 32, 34, 36, 37, 43, 46, 47], small for eight interventions [31, 33, 39, 43, 48, 50, 51], and medium for one intervention [49]. Data from Hinckson et al. [18] indicate a trivial effect size after 1 year of followup, but a medium effect size after 2 or 3 years. Henderson and colleagues' [38] SRTS intervention yielded a medium effect size for the morning trip and a trivial effect size for the afternoon trip. Data from Hunter et al. [39] indicated a medium decrease in AST as estimated with the swipe card methodology, but a small increase for self-reported AST. In Crawford and colleagues' [34] pilot program, there was a small effect size for the inner suburban school and a trivial one for the outer suburban school. In the 3group intervention by Ducheyne et al. [35], there was a small effect size when comparing the intervention and control groups, but a trivial effect size when comparing the intervention + parent (which targeted parents in addition to children) vs. the control group. Finally, data from McMinn et al. [44] suggest a small effect size for changes in minutes of moderate-to-vigorous PA per day, but a moderate effect size for changes in steps/day although both effect sizes were similar $(d=0.46$ and 0.52 respectively); however, effect size was trivial for changes in steps and MVPA during the school trip. Table 3 summarizes effect sizes by type of intervention; however, no clear pattern is evident.

\section{Moderators and mediators}

Thirteen studies examined potential moderators. Hinckson et al. $[17,18]$ noted that longer follow-up periods, smaller school size, higher school SES, and higher pre-intervention rate of AST predicted higher rates of AST at follow-up. Safe Routes to School interventions using multiple strategies (as defined by the 6P model) achieved larger increases in AST [42, 43], and a longer follow-up period was also associated with more substantial increases in AST [43]. In contrast, a short follow-up period was discussed as a potential reason for the lack of a significant mode shift in other interventions $[20,46]$. Mammen and colleagues [19] reported that parents of older students, those living closer to school and attending urban or suburban schools (relative to rural) were more likely to report "driving less" following the implementation of an STP. Of the potential moderators examined by Stewart et al. [48], only the percentage of students cycling at baseline was negatively associated with changes in cycling. In addition, Mendoza and colleagues' [45] results suggest that greater acculturation, more positive parental self-efficacy and outcome expectations may facilitate children's engagement in AST.

Goodman and colleagues [36] intended to assess children's participation in cycle training as a mediator of the relationship between exposure to the Bikeability program at the school level and children's cycling behavior. However they found a similar frequency of cycling among children exposed and unexposed to the program. No other study described formal mediation analyses.

\section{Discussion}

We have provided a comprehensive update on the effectiveness of AST interventions among children and adolescents. Our search strategy identified 27 papers, describing the findings of 30 distinct interventions, which have been published since the previous review [24]. Included interventions were quite diverse and changes in travel behaviors varied markedly across interventions. Included studies suggest that interventions with longer follow-up periods may achieve greater modal shifts. These observations are of particular importance for policymakers and practitioners implementing AST interventions.

Two large SRTS interventions found that interventions including both educational activities and infrastructure changes resulted in greater increases in AST than interventions using only one of these strategies $[42,43]$. These results are consistent with social-ecological models that posit that behavior is determined by multiple levels of influence including individual, interpersonal, community, policy and built environment factors [52, 53].

We noted that few interventions targeted secondary school students. As the correlates of AST may differ by age [54], one should not assume that interventions that are effective among children will work as well with adolescents. Adolescents generally have higher independent mobility [55] and, as such, the influence of parental perceptions on their school travel mode may be weaker. However, adolescents may have less favorable attitudes toward AST [56, 57], and this might be a key factor to address for interventions in secondary schools.

In the previous systematic review [24], all studies were rated as "weak" based on the EPHPP tool. In our review, $10 \%$ of the studies were rated "moderate" (even with a stricter interpretation of the blinding component of EPHPP) and, when the blinding component was dismissed as unfeasible, $30 \%$ of the studies were rated as "moderate" or "strong". This suggests a marginal improvement in study quality over the last 6 years; however the overall quality of evidence as assessed with the GRADE approach remains low. Our sensitivity analysis 
Table 3 Effect size of active school transportation interventions stratified by intervention type

\begin{tabular}{|c|c|c|}
\hline & Measure of effect size & Cohen's d \\
\hline \multicolumn{3}{|l|}{ Safe Routes to school } \\
\hline Henderson (2013) & Change in prevalence of AST (morning trip/afternoon trip) & $0.66 / 0.17$ \\
\hline McDonald (2014) & Change in prevalence of AST & 0.19 \\
\hline Østergaard (2015) & Change in number of weekly AST trips & 0.02 \\
\hline Stewart (2014) & Change in prevalence of AST & 0.28 \\
\hline \multicolumn{3}{|l|}{ School travel planning } \\
\hline Buliung (2011) & Change in prevalence of AST & 0.05 \\
\hline Crawford (2013) & $\begin{array}{l}\text { Change in prevalence of AST - inner suburban pilot school } \\
\text { (direct observation/hands-up survey) }\end{array}$ & $0.27 / 0.30$ \\
\hline Crawford (2013) & $\begin{array}{l}\text { Change in prevalence of AST - outer suburban pilot school } \\
\text { (direct observation/hands-up survey) }\end{array}$ & $-0.12 / 0.04$ \\
\hline Crawford (2013) & $\begin{array}{l}\text { Change in prevalence of AST in the program schools } \\
\text { (parent report/child report) }\end{array}$ & $0.04 /-0.06$ \\
\hline Hinckson (2011a) & Change in prevalence of AST & 0.14 \\
\hline Hinckson (2011b) & $\begin{array}{l}\text { Change in prevalence of AST according to length of follow-up } \\
\text { ( } 1 \text { year } / 2 \text { years } / 3 \text { years) }\end{array}$ & $-0.17 ; 0.51 ; 0.54$ \\
\hline Mammen (2014b) & Change in prevalence of AST (morning trip/afternoon trip) & $-0.02 ; 0.01$ \\
\hline \multicolumn{3}{|l|}{ Walking school buses } \\
\hline Mendoza (2011) & Change in percentage of trips using AST & 0.40 \\
\hline Sayers (2012) & Difference in $\%$ of time spent in MVPA & -0.32 \\
\hline \multicolumn{3}{|l|}{ Cycle training } \\
\hline Ducheyne (2014) & $\begin{array}{l}\text { Change in weekly time spent engaging in AST (intervention vs. } \\
\text { control group/intervention + parent vs. control group) }\end{array}$ & $0.46 / 0.03$ \\
\hline Johnson (2016) & $\begin{array}{l}\text { Difference in odds of cycling to school between trained and } \\
\text { untrained children (Bikeability survey) }\end{array}$ & 0.45 \\
\hline Johnson (2016) & $\begin{array}{l}\text { Difference in odds of cycling to school between trained and } \\
\text { untrained (CensusAtSchool survey) }\end{array}$ & 0.26 \\
\hline Goodman (2016) & $\begin{array}{l}\text { Difference in odds of cycling to school between trained and } \\
\text { untrained (school level/individual level) }\end{array}$ & $-0.17 ; 0.18$ \\
\hline \multicolumn{3}{|l|}{ Special events } \\
\hline Bungum (2014) & Change in number of students engaging in AST & 0.29 \\
\hline Coombes (2016) & $\begin{array}{l}\text { Change in proportion of trips using AST at 7-week and } \\
\text { 20-week follow ups respectively }\end{array}$ & $-0.32 ; 0.24$ \\
\hline Hunter (2015) & $\begin{array}{l}\text { Change in prevalence of AST (measured with swipe } \\
\text { card/self-report) }\end{array}$ & $-0.61 ; 0.34$ \\
\hline \multicolumn{3}{|c|}{ Multi-component interventions } \\
\hline Christiansen (2014) & Change in odds of engaging in AST & 0.13 \\
\hline Xu (2015) & Change in odds of engaging in AST & 0.45 \\
\hline \multicolumn{3}{|c|}{ Curriculum-based interventions } \\
\hline McMinn (2012) & $\begin{array}{l}\text { Difference in commuting steps and MVPA between } \\
\text { intervention and control groups }\end{array}$ & $0.06 /-0.03$ \\
\hline McMinn (2012) & $\begin{array}{l}\text { Difference in daily steps and MVPA between } \\
\text { intervention and control groups }\end{array}$ & $0.52 / 0.46$ \\
\hline Villa-Gonzalez (2016) & Changes in weekly number of active trips & 0.40 \\
\hline \multicolumn{3}{|l|}{ Drop-off spots } \\
\hline Vanwolleghem (2014) & Change in frequency of AST & 0.75 \\
\hline \multicolumn{3}{|l|}{ Crossing guards } \\
\hline Gutierrez (2014) & Change in number of students engaging in AST & 0.03 \\
\hline
\end{tabular}

AST active school transportation, MVPA moderate-to-vigorous physical activity. Effect sizes were computed as detailed in Additional file 2. Some studies appear more than once because they have multiple measures of effect size. Cohen's $d$ could not be computed for 5 interventions because insufficient information was provided by the authors. Following Cohen's ${ }^{28}$ guidelines, effect size can be categorized as trivial $(d<0.2)$, small $(d=0.2)$, medium $(d=0.5)$, or large $(d=0.8)$ 
shows that the blinding component exerted a floor effect on quality scores. Because all interventions received a "weak" rating for blinding, they could not be rated higher than "moderate". Future improvement in quality ratings could be made by controlling for confounders and by using valid and reliable measures of AST, which have been reviewed elsewhere [58].

The calculated effect sizes for most interventions were trivial to small based on Cohen's [28] thresholds. Although these widely-used thresholds are arbitrary, we have used them in the absence of alternative options. Given the large reach of interventions such as SRTS and STP, an effect size labeled as "trivial-to-small" may still be highly relevant from a population health perspective. Interestingly, a pooled intervention effect of $d=0.12$ was obtained in a meta-analysis of 30 controlled trials on PA interventions among children and adolescents [59].

Furthermore, while our review focused specifically on the effect of interventions on travel behaviors, some included interventions have documented positive changes in other important outcomes such as children's cycling skills [35], safe street crossing behaviors [37], attitudes toward AST [40], and higher daily PA [44, 45]. Substantial reductions in road traffic injuries among children have also been noted following implementation of SRTS [15]. More broadly, it has been proposed that interventions such as SRTS may benefit the larger communities in which they are implemented, and not only children [60].

\section{Mediators and moderators}

A better understanding of the mediators and moderators of AST interventions could help identify what works for whom and why [61, 62]. Of particular interest, many studies emphasized the importance of having long term follow-ups given that implementation of complex AST interventions may require a substantial amount of time [17-20, 43, 46]. Similarly, qualitative evaluations focusing on the implementation of AST interventions also identify lack of time as a key challenge [63, 64]. To address the issue of follow-up length, some authors suggested that granting agencies should be encouraged to provide more long term funding $[63,64]$.

While there has been increased interest in studying moderators of AST interventions, none of the included studies conducted formal mediation analyses and most interventions did not include an explicit theoretical framework. Given the important role of parents in travel mode decision making [65], interventions that increase road safety may be more effective if they also target parents' self-efficacy in allowing their child to engage in AST [45].

\section{Implementation of interventions}

Understanding the implementation of complex AST interventions may provide valuable information for the reader to contextualize the effectiveness of such interventions. This may be particularly important for interventions such as SRTS and STP that are essentially evaluated as "natural experiments" [66] because in most cases, exposure to the intervention is not under the control of the investigators. This is a threat to internal validity because the fidelity of implementation varies, but at the same time, it represents more closely how an intervention is implemented in the "real world". Many interventions included in this review reported that implementation varied substantially between schools $[19,20,32,34,46]$, and in some cases, planned changes were not implemented as scheduled [32, 37, 46]. Crawford and Garrard [34] also reported that the implementation of the Ride2School program was affected by the motivation of school communities. Such challenges and discrepancies may bias our results toward the null hypothesis.

Lack of resources or unequal access to resources has been noted by many authors as a limitation to AST interventions [32, 63, 64]. In Canada, STPs and WSBs are implemented by non-governmental organizations and lack of support from provincial and federal governments has been identified as a major barrier [64]. In Texas, stakeholders expressed difficulty in navigating the SRTS regulatory process and emphasized that access to SRTS funding was very challenging for low income communities given that no up-front funding was provided [63]. More generally, WSBs typically rely on volunteers which often makes long term sustainability challenging [23, 67]. Providing paid WSB leaders may help overcome this issue.

\section{Strengths and limitations}

As in the previous review [24], we noted that many included studies did not include a control group. Another limitation is that the original EPHPP tool seems better suited to assess studies where the unit of allocation is the individual. To address this issue, we have modified the tool so that the questions are more relevant to school-based interventions (see Additional file 1). Nevertheless, like other quality assessment tools, the scoring system of the EPHPP is rigid and may not always distinguish more robust studies from weaker ones [68]. For example, in our review, no study reported that outcome assessors were blinded, creating a floor effect whereby no intervention can be rated higher than "moderate". Notwithstanding the importance of blinding in preventing observer bias and Hawthorne effects, a quality assessment tool should be able to discriminate stronger studies from weaker ones. Our sensitivity analysis without the blinding component of the EPHPP intended to address this issue. We acknowledge that the use of a different quality assessment tool could have resulted in different ratings of study quality as observed previously [68]. Finally, the large heterogeneity in the measurement 
and operationalization of AST precluded meta-analysis. The development of a standard measurement protocol may help address this issue.

The rigorous systematic review process is an important strength of the study. We followed the same search strategy as Chillón and colleagues [24] and computed standardized effect sizes which should help readers interpret the effectiveness of interventions and perform sample size calculations. Finally, the discussion of moderators, mediators and factors related to implementation should help researchers refine current interventions.

\section{Conclusions}

The present systematic review highlights the diversity of interventions that have been implemented to promote AST in the last few years, and shows that travel behavior change varied markedly between interventions. Many interventions have shown significant increases in AST, but caution is required in interpretation given the low quality of evidence. This underscores a need for interventions using stronger study designs.

Our findings have implications for researchers and practitioners. First, it may take time for interventions to have an effect on children's travel behaviors. Therefore, follow-ups of at least 2 years should be conducted when possible to minimize the risk of type II error. Second, while many authors indicated that implementation of interventions varied markedly across schools, it is unclear how this variation may influence effectiveness. Hence future research should examine the potential moderating effect of implementation. The fact that some interventions were not implemented as planned suggests that some of the effect sizes reported herein may be conservative. Third, there remains a clear need for investigation of the mediators of travel behavior change.

Only three interventions included some high schools, highlighting a need for more research intervening in secondary school settings. This is important given that the factors associated with AST may differ markedly between children and adolescents. Finally, because some children may live too far from their school, interventions aiming to promote active transportation to/from other destinations such as parks, shops, sport venues, and friends' and relatives' houses may also be warranted [69].

\section{Additional files}

Additional file 1: Appendix 1. Adjusted criteria for the Effective Public Health Practice Project quality assessment tool for quantitative studies. (DOCX $33 \mathrm{~kb}$ )

Additional file 2: Appendix 2. Computation of effect sizes. (DOCX 34 kb)

\section{Abbreviations}

AST: Active school transport; EPHPP: Effective public health practice project; GRADE: Grades of recommendation, assessment, development, and evaluation; PA: Physical activity; SRTS: Safe routes to school; STP: School travel plans; UK: United Kingdom; US: United States; WSB: Walking school buses

\section{Acknowledgements}

Not applicable.

\section{Funding}

GF holds a Canadian Institutes of Health Research-Public Health Agency of Canada (CIHR-PHAC) Chair in Applied Public Health. RL was supported by a postdoctoral fellowship from the Canadian Institutes of Health Research. Funders had no role in the study.

Availability of data and materials

The data that was used to compute effect sizes is available in Additional file 2.

\section{Authors' contributions}

$\mathrm{RL}$ completed quality assessment, data extraction, statistical analyses, and drafted the manuscript. GM conducted the search, screened papers for inclusion, assisted in data extraction and provided feedback on the manuscript. DAR conducted quality assessment, assisted with statistical analyses, and provided feedback on the manuscript. GF screened papers for inclusion and provided feedback on the manuscript. All authors read and approved the final manuscript.

Ethics approval and consent to participate

Not applicable.

Consent for publication

Not applicable.

Competing interests

The authors declare that they have no competing interests.

\section{Publisher's Note}

Springer Nature remains neutral with regard to jurisdictional claims in published maps and institutional affiliations.

\section{Author details}

${ }^{1}$ Healthy Active Living and Obesity Research Group, Children's Hospital of Eastern Ontario Research Institute, Ottawa ON K1H 8L1, Canada. ${ }^{2}$ Faculty of Health Sciences University of Lethbridge, 4401 University Drive, office M3049 Lethbridge, Alberta T1K 3M4, Canada. ${ }^{3}$ Centre for Addiction and Mental Health, Institute for Mental Health Policy Research, 1001 Queen St West, Toronto, ON M6J 1H4, Canada. ${ }^{4}$ School of Psychological Sciences and Health, University of Strathclyde, 16 Richmond St, Glasgow G1 1XQ, Glasgow, UK. ${ }^{5}$ School of Kinesiology, University of British Columbia, D H Copp Building 4606, 2146 Health Sciences Mall, Vancouver, BC V6T 1Z3, Canada. ${ }^{6}$ Center for Hip Health and Mobility, Robert H.N. Ho Research Centre, 5th Floor, 2635 Laurel St, Vancouver, BC V5Z 1M9, Canada.

Received: 28 April 2017 Accepted: 20 December 2017

Published online: 01 February 2018

References

1. Larouche R, Saunders TJ, Faulkner GEJ, Colley RC, Tremblay MS. Associations between active school transport and physical activity, body composition and cardiovascular fitness: a systematic review of 68 studies. J Phys Act Health. 2014;11(1):206-27.

2. Schoeppe S, Duncan MJ, Badland H, Oliver M, Curtis C. Associations of children's independent mobility and active travel with physical activity, sedentary behaviour, and weight status: a systematic review. J Sci Med Sport. 2013;16(4):312-9.

3. Andersen LB, Wedderkopp N, Kristensen P, Moller NC, Froberg K, Cooper AR. Cycling to school and cardiovascular risk factors: a longitudinal study. J Phys Act Health. 2011:8(8):1025-33.

4. de Nazelle A, Nieuwenhuijsen MJ, Antó JM. Improving health through policies that promote active travel: a review of evidence to support integrated health impact assessment. Environ Int. 2011;37(4):766-77. 
5. Marshall JD, Wilson RD, Meyer KR, Rajangam SK, McDonald NC, Wilson EJ. Vehicle emissions during children's school commuting: impacts of education policy. Env Sci Technol. 2010;44(5):1537-43.

6. Ramanathan S, O'Brien C, Faulkner G, Stone M. Happiness in motion: emotions, well-being and active school travel. J School Health. 2014;84(8):516-23.

7. Rissotto A, Tonucci F. Freedom of movement and environmental knowledge in elementary school children. J Environ Psychol. 2002;22:65-77.

8. Martinez-Gomez D, Ruiz JR, Gomez-Martinez S, et al. Active commuting to school and cognitive performance in adolescents. Arch Pediatr Adolesc Med. 2011;165(4):300-5.

9. Gray C, Larouche R, Barnes JD, et al. Are we driving our kids to unhealthy habits? Results from the active healthy kids Canada 2013 report card on physical activity for children and youth. Int J Environ Res Public Health. 2014;11(6):6009-20.

10. Grize $L$, Bringolf-Isler B, Martin E, Braun-Farhländer C. Trend in active transportation to school among Swiss school children and its associated factors: three cross-sectional surveys 1994, 2000 and 2005. Int J Behav Nutr Phys Act. 2010;7:28.

11. McDonald NC. Active commuting to school: trends among US schoolchildren 1969-2001. Am J Prev Med. 2007;32(6):509-16.

12. Trang NHHD, Hong TK, Dibley MJ. Active commuting to school among adolescents in ho chi Minh City, Vietnam: changes and predictors in a longitudinal study, 2004 to 2009. Am J Prev Med. 2012;42(2):120-8.

13. Tremblay MS, Gray CE, Akinroye KK, et al. Physical activity of children: a global matrix of grades comparing 15 countries. J Phys Act Health. 2014; 11(Suppl 1):113-25.

14. Safe Routes to School National Partnership. National Policy and Advocacy. 2015. Available from: http://www.saferoutespartnership.org/blog/6-es-saferoutes-school-embracing-equity Accessed 11 Feb 2017.

15. DiMaggio C, Li G. Effectiveness of a safe routes to school program in preventing school-aged pedestrian injury. Pediatrics. 2013;131(2):290-6.

16. Muennig PA, Epstein M, Li G, DiMaggio C. The cost-effectiveness of new York City's safe routes to school program. Am J Public Health. 2014;104(7): 1294-9.

17. Hinckson EZ, Badland HM. School travel plans: preliminary evidence for changing school-related travel patterns in elementary school children. Am J Health Promot. 2011;25(6):368-71

18. Hinckson EA, Garrett N, Duncan S. Active commuting to school in New Zealand children (2004-2008): a quantitative analysis. Prev Med. 2011;52(5):332-6.

19. Mammen G, Stone MR, Faulkner G, et al. Active school travel: an evaluation of the Canadian school travel planning intervention. Prev Med. 2014;60:55-9.

20. Mammen G, Stone MR, Buliung R, Faulkner G. "Putting school travel on the map": facilitators and barriers to implementing school travel planning in Canada. J Transp Health. 2015;2(3):318-26.

21. Rowland D, DiGuiseppi C, Gross M, Afolabi E, Roberts I. Randomised controlled trial of site specific advice on school travel patterns. Arch Dis Child. 2003:88:8-11.

22. Kingham S, Ussher S. An assessment of the benefits of the walking school bus in Christchurch, New Zealand. Transp Res Part A-Policy Pract. 2007;41 (6):502-10.

23. Smith L, Norgate SH, Cherrett T, Davies N, Winstanley C, Harding M. Walking school buses as a form of active transportation for children - a review of the evidence. J Sch Health. 2015;85:197-210.

24. Chillón P, Evenson KR, Vaughn A, Ward DS. A systematic review of interventions for promoting active transportation to school. Int J Behav Nutr Phys Act. 2011:8(10)

25. Effective Public Health Practice Project. Quality Assessment Tool for Quantitative Studies. 2009. Available from http://www.ephpp.ca/tools.html Accessed 11 Feb 2017

26. Guyatt $\mathrm{GH}, \mathrm{Oxman} A D$, Vist GE, et al. GRADE: an emerging consensus on rating quality of evidence and strength of recommendations. BMJ. 2008;336:924-6.

27. Guyatt GH, Oxman AD, Vist GE, Kunz R, Falck-Ytter $Y$, Schünemann HJ. GRADE: what is "quality of evidence" and why is it important to clinicians? BMJ. 2008;336:995-8.

28. Cohen J. Statistical power analysis for the behavioral science. 2nd ed. Hillsdale, NJ: Lawrence Erlbaum; 1988.

29. Buckley A, Lowry MB, Brown H, Barton B. Evaluating safe routes to school events that designate days for walking and bicycling. Transp Policy. 2013;30: 294-300.

30. Buliung R, Faulkner G, Beesley T, Kennedy J. School travel planning: mobilizing school and community resources to encourage active school transportation. J Sch Health. 2011:81:704-12.
31. Bungum TJ, Clark S, Aguilar B. The effect of an active transport to school intervention at a suburban elementary school. Am J Health Educ. 2014;45(4): 205-9.

32. Christiansen LB, Toftager M, Ersbø\|ll AK, Troelsen J. Effects of a Danish multicomponent physical activity intervention on active school transport. J Transp Health. 2014;1:174-81.

33. Coombes $\mathrm{E}$, Jones A. Gamification of active travel to school: a pilot evaluation of the beat the street physical activity intervention. Health Place. 2016;39:62-9.

34. Crawford S, Garrard J. A combined impact-process evaluation of a program promoting active transport to school: understanding the factors that shaped program effectiveness. J Environ Public Health. 2013;816961

35. Ducheyne F, de Bourdeaudhuij I, Lenoir M, Cardon G. Effects of a cycle training course on children's cycling skills and levels of cycling to school. Accid Anal Prev. 2014:67:49-60.

36. Goodman A, van Sluijs EM, Ogilvie D. Impact of offering cycle training in schools upon cycling behaviour: a natural experimental study. Int J Behav Nutr Phys Act. 2016;13:34.

37. Gutierrez CM, Slagle D, Figueras K, Anon A, Huggins AC, Hotz G. Crossing guard presence: impact on active transportation and injury prevention. J Transp Health. 2014:1:116-23.

38. Hendersen S, Tanner R, Klanderman S, Mattera A, Webb LM, Steward J. Safe routes to school: a public health practice success story-Atlanta, 20082010. J Phys Act Health. 2013;10(2):141-2.

39. Hoelscher D, Ory M, Dowdy D, et al. Effects of funding allocation for safe routes to school programs on active commuting to school and related behavioral, knowledge, and psychosocial outcomes: results from the Texas childhood obesity prevention policy evaluation (T-COPPE) study. Environ Behav. 2016;48(1):210-29.

40. Hunter RF, de Silva D, Reynolds V, Bird W, Fox KR. International inter-school competition to encourage children to walk to school: a mixed methods feasibility study. Int J Behav Nutr Phys Act. 2015:8:19.

41. Johnson R, Frearson M, Hewson P. Can bicycle training for children increase active travel? Eng Sustain. 2016;169(2):49-57.

42. McDonald NC, Yang Y, Abbott SM, Bullock AN. Impact of the safe routes to school program on walking and biking: Eugene. Oreg Study Transp Policy. 2013;29:243-8

43. McDonald NC, Steiner RL, Lee C, Smith TR, Zhu X, Yang Y. Impact of the safe routes to school program on walking and bicycling. J Am Plan Assoc. 2014;80(2):153-67.

44. McMinn D, Rowe DA, Murtagh S, Nelson NM. The effect of a school-based active commuting intervention on children's commuting physical activity and daily physical activity. Prev Med. 2012;54:316-8.

45. Mendoza JA, Watson K, Baranowski T, Nicklas TA, Uscanga DK, Hanfling MJ. The walking school bus and children's physical activity: a pilot cluster randomized controlled trial. Pediatrics. 2011;128(3):e537-44.

46. Østergaard L, Støckel JT, Andersen LB. Effectiveness and implementation of interventions to increase commuter cycling to school: a quasi-experimental study. BMC Public Health. 2015;15:1199.

47. Sayers SP, LeMaster JW, Thomas IM, Petroski GF, Ge B. A walking school bus program: impact on physical activity in elementary school children in Columbia, Missouri. Am J Prev Med. 2012;43(5S4):S384-9.

48. Stewart O, Moudon AV, Claybrooke C. Multistate evaluation of safe routes to school programs. Am J Health Promot. 2014;28(3S):S89-96.

49. Vanwolleghem G, D'Haese S, Van Dyck D, de Bourdeaudhuij I, Cardon G Feasibility and effectiveness of drop-off spots to promote walking to school. Int J Behav Nutr Phys Act. 2014;11:136.

50. Villa-González E, Ruiz JR, Ward DS, Chillón P. Effectiveness of an active commuting school-based intervention at 6-month follow-up. Eur J Pub Health. 2016;26(2):272-6.

51. Xu F, Ware RS, Leslie E, Tse LA, Wang Z, Li J, Wang Y. Effectiveness of a randomized controlled lifestyle intervention to prevent obesity among Chinese primary school students: CLICK-obesity study. PLoS One. 2015; 10(10):e0141421.

52. Giles-Corti B. People or places: what should be the target? J Sci Med Sport. 2006:9(5):357-66

53. Sallis JF, Cervero RB, Ascher W, Henderson KA, Kraft MK, Kerr J. An ecological approach to creating active living communities. Ann Rev Public Health. 2006;27:297-322.

54. Mitra R, Buliung RN. Exploring differences in school travel mode choice behaviour between children and youth. Transp Policy. 2015;42:4-11. 
55. Shaw B, Bicket M, Elliot B, Fagan-Watson B, Mocca E, Hillman M. Children's independent mobility: an international comparison and recommendations for action. London, UK: Policy Studies Institute; 2015.

56. Lorenc T, Brunton G, Oliver S, Oliver K, Oakley A. Attitudes to walking and cycling among children, young people and parents: a systematic review. J Epidemiol Community Health. 2008;62:852-7.

57. Stuckless J. Project overview: high school pilot project 2010-2012. Green Communities Canada. 2012. Available from: http://activesafe. nonprofitwebsites.ca/sites/default/files/Overview\%20of\%200ttawa\%20High \%20School\%20STP\%20Pilot\%20Project\%20-\%20Jamie\%20Stuckless.pdf Accessed 11 Feb 2017

58. Larouche R, Oyeyemi AL, Prista A, Onywera VO, Akinroye KK, Tremblay MS. A systematic review of active transportation research in Africa and the psychometric properties of measurement tools in children and youth. Int J Behav Nutr Phys Act. 2014:11:129.

59. Metcalf B, Henley W, Wilkin T. Effectiveness of intervention on physical activity of children: systematic review and meta-analysis of controlled trials with objectively measured outcomes (EarlyBird 54). BMJ. 2012;345:e5888.

60. Watson M, Dannenberg AL. Investments in Safe Routes to school projects: public health benefits for the larger community. Prev Chronic Dis. 2008;5(3):A90.

61. Baranowski T, Anderson C, Carmack C. Mediating variable framework in physical activity interventions: how are we doing? How might we do better? Am J Prev Med. 1998;15(4):266-97.

62. Bauman AE, Sallis JF, Dzewaltowski DA, Owen N. Toward a better understanding of the influences on physical activity: the role of determinants, correlates, causal variables, mediators, moderators, and confounders. Am J Prev Med. 2002;23(Suppl 1):5-14.

63. Atteberry H, Dowdy D, Oluyomi A, Nichols D, Ory MG, Hoelscher DM. A contextual look at safe routes to school implementation in Texas. Env Behav. 2016:48(1):192-209.

64. Mammen G, Stone MR, Buliung N, Faulkner G. School travel planning in Canada: identifying child, family, and school-level characteristics associated with travel mode shift from driving to active school travel. J Transp Health. 2014;1(4):288-94

65. Faulkner GEJ, Richichi V, Buliung RN, Fusco C, Moola F. What's "quickest and easiest?": parental decision making about school trip mode. Int J Behav Nutr Phys Act. 2010;7:62.

66. Ramanathan S, Allison KR, Faulkner G, Dwyer JJM. Challenges in assessing the implementation and effectiveness of physical activity and nutrition policy interventions as natural experiments. Health Promot Int. 2008;23(3): 290-7.

67. Kingham S, Ussher S. Ticket to a sustainable future: an evaluation of the long-term durability of the walking school bus programme in Christchurch, New Zealand. Transp Policy. 2005;12(4):314-23.

68. Armijo-Olivo S, Stiles CR, Hagen NA, Blondo PD, Cummings GG. Assessment of study quality for systematic reviews: a comparison of the Cochrane collaboration risk of bias tool and the effective public health practice project quality assessment tool: methodological research. J Eval Clin Pract 2012:18(1):12-8.

69. Larouche R, Barnes J, Tremblay MS. Too far to walk or bike? Can J Public Health. 2013:104(7):e487-9.

\section{Submit your next manuscript to BioMed Central and we will help you at every step:}

- We accept pre-submission inquiries

- Our selector tool helps you to find the most relevant journal

- We provide round the clock customer support

- Convenient online submission

- Thorough peer review

- Inclusion in PubMed and all major indexing services

- Maximum visibility for your research

Submit your manuscript at www.biomedcentral.com/submit

) Biomed Central 